- Case Report

\title{
Persistent Shoulder Pain in Young Male: Osteosarcoma
}

\author{
Punitha Arinima ${ }^{1, *}$, Azlina Ishak ${ }^{2}$ \\ ${ }^{1}$ Kota Bharu Health Centre, Kelantan, Malaysia \\ ${ }^{2}$ Department of Family Medicine, Universiti Sains Malaysia, Kelantan, Malaysia
}

\begin{abstract}
A 16-year-old Malay boy presented to Kota Bharu Health Care Centre, Kelantan, with left shoulder pain after sustaining a fall. On further history taking, it was noted that the pain preceded the fall by 1 month. The early changes of osteosarcoma were visible on an X-ray during the initial presentation; however, this was missed by the primary care doctors. Three months later, the patient presented with persistent pain in the left shoulder and was diagnosed with osteosarcoma.
\end{abstract}

Keywords: Osteosarcoma; Shoulder Pain; Shoulder Swelling 


\section{INTRODUCTION}

Bone cancers or sarcomas are divided into three types: osteosarcoma, Ewing sarcoma, and chondrosarcoma. They can also be divided into primary and secondary tumors of the bone. Primary tumors are derived from the bone itself, whereas secondary tumors are metastases of cancer from other parts of the body. Osteosarcoma is the most common but also a very rare type of primary malignant bone tumor. Despite being the most common bone tumor, it is not fully understood. The World Health Organization classified osteosarcoma as osteogenic and cartilage tumors. It predominantly affects male patients $<20$ years of age. However, a small proportion of cases occurs in elderly individuals $>50$ years of age. The most common sites of osteosarcoma are the long bones such as the humerus, femur, and tibia. Nevertheless, the craniofacial bones, pelvis, and ribs can also be affected.

Bone pain and swelling are the typical presentations of bone cancer. Some patients present with fracture and dislocation of the involved joints. Due to its rare nature, it is easy for doctors at the primary care level to dismiss complaints of pain and treat it symptomatically, thereby innocently missing the diagnosis of bone tumors. Unfortunately, this delays the diagnosis of osteosarcoma, leading to the inability to properly initiate treatment.

This case report reviews an unfortunate case demonstrating the learning curve for doctors at primary care centers and urges them to be more alert to and vigilant of the early symptoms of osteosarcoma.

\section{CASE REPORT}

A 16-year-old Malay boy presented to Kota Bharu Health Care Centre, Kelantan, after sustaining a fall at home on a slippery floor. He fell on his left side and hit his left shoulder. Post-trauma, the patient complained of pain and swelling of his left shoulder. The range of move-

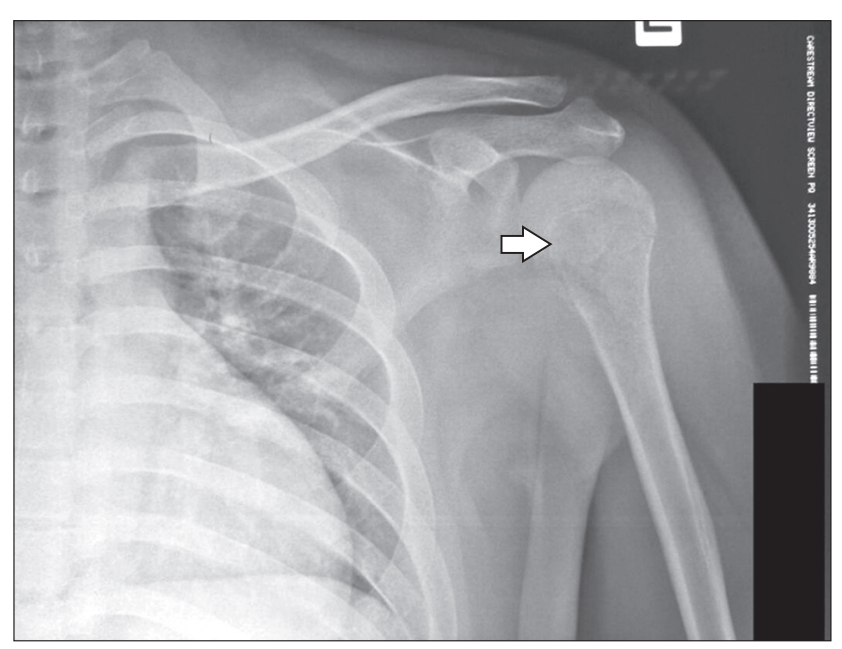

Figure 1. X-ray of left shoulder taken during the patient's first visit to Kota Bharu Health Care Centre. A lytic lesion (arrow) with a cortical break at the medial aspect of the left humeral neck is visible. No periosteal reaction or soft-tissue component is visible. ments of his left shoulder was reduced due to pain. However, the doctor at the health care center reported that the X-ray of his left shoulder (Figure 1) was normal with no fractures or dislocations. Despite given adequate analgesics, the patient's left shoulder continued to swell and cause increasing pain.

One month after the initial presentation, he re-visited the health care center with more obvious swelling and worsening pain of his left shoulder. He had tried massage therapy by local masseurs but reported no improvement. On that second visit, a repeat X-ray of his left shoulder revealed no new findings. Two weeks later, a physiotherapist diagnosed left anterior shoulder dislocation. A closed manual reduction was unsuccessful. The patient was then referred to the orthopedic

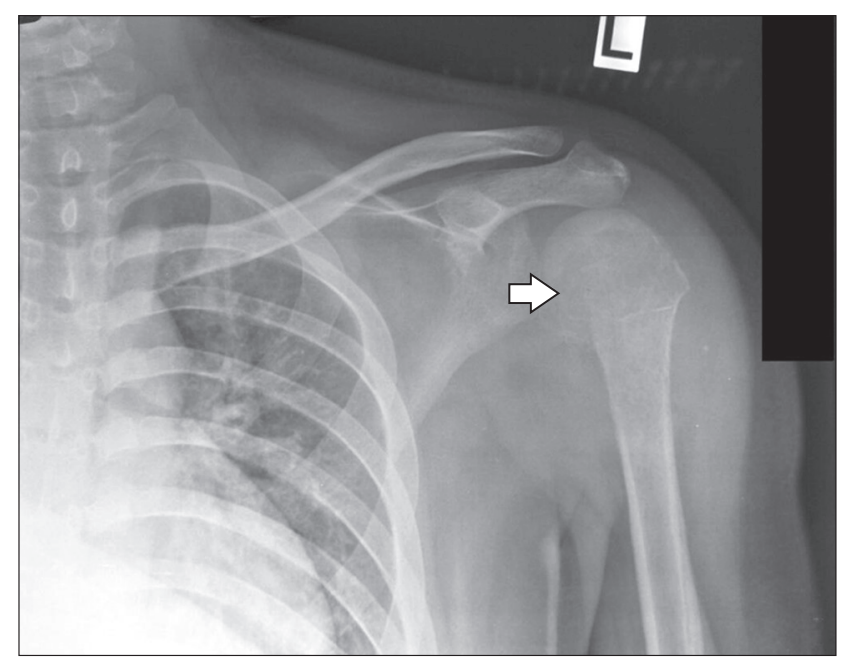

Figure 2. X-ray of left shoulder taken 1 month later. The lytic lesion (arrow) is larger than the one of the $\mathrm{X}$-ray taken 1 month prior. This is associated with a periosteal reaction. There is now evidence of a pathological fracture at the humeral neck.

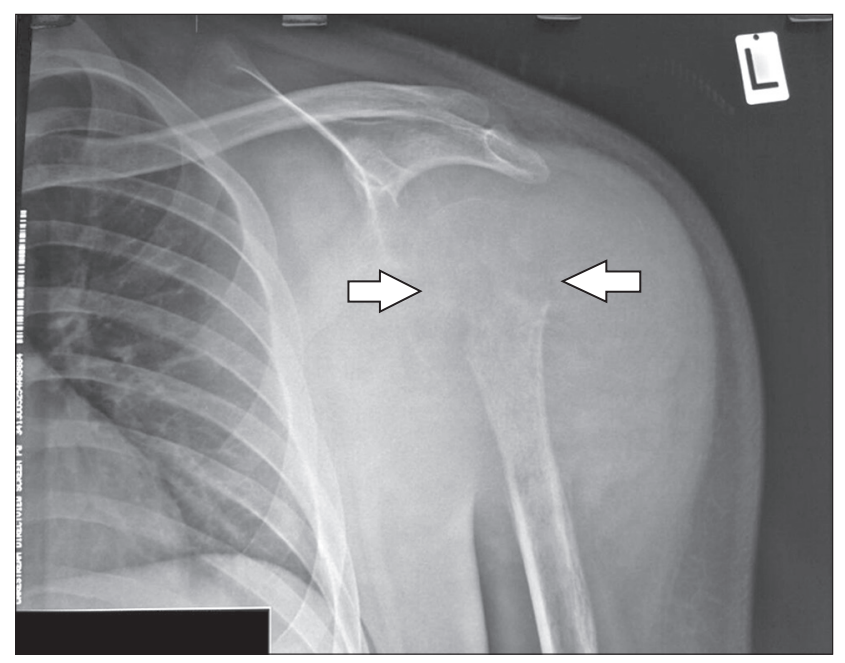

Figure 3. X-ray of the left shoulder taken 3 months after the initial presentation. A Iytic lesion (arrows) is visible at the proximal end of the left humerus with a wide zone of transition and resorption of the humeral head. Codman's triangle is also visible, and a periosteal reaction is visible to the mid-shaft of the humerus. 
team at Hospital Raja Perempuan Zainab II for open reduction and screw fixation. He refused this intervention.

Three months after the first presentation, the patient re-visited Kota Bharu Health Care Centre for the third time as the pain and swelling of his left shoulder had become intolerable. No fever or constitutional symptoms were noted. On further questioning, the patient revealed that the pain in his left shoulder had started 1 month before his fall, but the originally mild intensity was worsened by the fall.

The patient had no significant past medical, surgical, allergy, or family history. He had stopped schooling and was unemployed. He was also a non-smoker. His general examinations were unremarkable. His blood pressure was 118/74 mm Hg, pulse was 120 beats per minute, respiration rate was 22 breaths per minute; he was afebrile; he had a pain score of 2/10; and his oxygen saturation was $100 \%$ on room air. Examination of his left shoulder revealed diffuse swelling of the left shoulder extending to just above his left elbow with no changes in skin color. No wounds were present. On palpation, diffuse tenderness was detected at the proximal portion of the left humerus. Range of motion of the shoulder was almost completely limited due to pain. There was no remarkable difference between active and passive movement. Despite reduced sensation at the axillary nerve distribution, the motor and sensory aspects of the radial, ulnar, and median nerves were intact. The radial pulse at the left wrist was palpable with good volume. Findings of examinations of the respiratory, cardiovascular, and gastrointestinal systems were insignificant. However, a lymph node measuring $2 \times 3 \mathrm{~cm}$ was palpable in his left supraclavicular region with a firm to hard consistency. No other lymph nodes were palpable.

Complete blood count, renal profile, and liver function test results were normal. An X-ray of the left shoulder (Figures 2, 3) revealed softtissue swelling at the shoulder. A lytic lesion was seen at the proximal end of the humerus with a wide zone of transition and resorption of the humeral head. Codman's triangle was also noted as well as a periosteal reaction was noted until the mid-shaft of the humerus.

\section{DISCUSSION}

Approximately $6 \%$ of all childhood malignancies are malignant bone tumors, the two most frequently encountered being osteosarcoma and Ewing sarcoma. Osteosarcoma originates from primitive bone-forming mesenchymal cells and is the most common primary bone malignancy. Although its incidence is equal among races, the incidence by sex has rarely been reported. While it is commonly seen in males, ${ }^{1)}$ sex is not associated with the incidence of osteosarcoma. ${ }^{2)}$ There is a strong correlation between young age $(<20$ years of age) and the incidence of osteosarcoma. ${ }^{1,2)}$ As the pubertal growth spurt occurs at this age, it is postulated that the occurrence of osteosarcoma is related to rapid growth. The long bones of the extremities, especially close to the metaphyseal growth plates, are the most common sites of osteosarcoma, but it can occur in other sites, such as the skull, jaw, and pelvis. It is predominant at the femur $(42 \%$, with $75 \%$ in the distal femur), followed by the tibia ( $19 \%$, with $80 \%$ in the proximal tibia) and the hu- merus (10\%, with $90 \%$ in the proximal humerus). ${ }^{1)}$ Despite a reduction in the death rates of osteosarcoma of approximately $1.3 \%$ per year, the overall 5-year survival rate is $68 \%$.

There is variation in the presentation of bone cancer, of which bone pain is the main reason for patients to seek medical attention. ${ }^{3)}$ Constitutional symptoms and lymph node metastasis are less common in osteosarcoma. Age $\geq 64$ years; female sex; undifferentiated pleomorphic sarcoma, osteosarcoma, or chondrosarcoma; increased tumor depth; and primary tumor size are the risk factors of lymph node metastasis, which significantly reduces patient 5 -year survival. ${ }^{4}$ A detailed history taking is crucial to diagnosis. In the case above, the earlier primary care practitioner failed to identify the cardinal sign of osteosarcoma, probably overlooking the injury that would have caused the pain.

Song et al. ${ }^{5)}$ reported on 10 cases of osteosarcomas that were missed by primary physicians during the early presentation. It was discovered that the plain radiologic findings of incipient-stage osteosarcoma include trabecular destruction (100\%), cortical disruption (60\%), periosteal reaction $(60 \%)$, and soft tissue mass (10\%). Intramedullary matrix changes were osteosclerosis in six and osteolysis in four patients. On progression, four cases with minimal sclerosis changed to osteoblastic lesion in three and osteolytic lesion in one. Four cases with faint osteolytic foci transformed into osteolytic lesion in three and a mixed pattern in one. A shoulder X-ray of the patient showed early changes induced by osteosarcoma, which again was missed at the primary care.

It is important that primary care physicians, who serve as gatekeepers to various specialties, have reasonable depth of knowledge to diagnose and treat patients. Moreover, well-written criteria for referral to certain subspecialties would be helpful in the early detection of rare diseases and further reduce referral delay. The Danish Cancer Patient Pathway defines a set of alarming symptoms as criteria for referral to a sarcoma center, which includes a soft-tissue tumor $>5 \mathrm{~cm}$ or a deepseated, fast-growing soft-tissue tumor, palpable bone tumor, and deep persistent bone pain. It noted that 179 (69\%) of 258 sarcoma patients were referred with alarming symptoms, while the remaining 79 sarcoma patients who were referred without alarming symptoms. Of them, seven were found accidentally on imaging, five were referred with suspected recurrence of a sarcoma, 64 were referred with a confirmed histological diagnosis, and three were referred for other reasons. ${ }^{6)}$ It was also noted that the sending of histological samples by primary care doctors delays the patient referral and the diagnosis of osteosarcoma.

A high index of suspicion is mandatory for any bone pain with or without changes on radiological imaging. Early referral and choosing the right imaging modality is vital for the diagnosis and management of patients with suspected sarcomas. ${ }^{7)}$

\section{CONFLICT OF INTEREST}

No potential conflict of interest relevant to this article was reported. 


\section{REFERENCES}

1. Ottaviani G, Jaffe N. The epidemiology of osteosarcoma. Cancer Treat Res 2009;152:3-13.

2. Ferrari A, Dirksen U, Bielack S. Sarcomas of soft tissue and bone. Prog Tumor Res 2016;43:128-41.

3. Buvarp Dyrop H, Vedsted P, Rædkjær M, Safwat A, Keller J. Routes to diagnosis for suspected sarcoma: the impact of symptoms and clinical findings on the diagnostic process. Sarcoma 2016;2016:8639272.

4. Vasiliev NV. Lymph node metastasis of osteosarcomas. Arkh Patol 2016;78:58-63.
5. Song WS, Jeon DG, Cho WH, Kong CB, Cho SH, Lee JW, et al. Plain radiologic findings and chronological changes of incipient phase osteosarcoma overlooked by primary physicians. Clin Orthop Surg 2014;6: 230-5.

6. Dyrop HB, Vedsted P, Rædkjær M, Safwat A, Keller J. Imaging investigations before referral to a sarcoma center delay the final diagnosis of musculoskeletal sarcoma. Acta Orthop 2017;88:211-6.

7. Ilaslan H, Schils J, Nageotte W, Lietman SA, Sundaram M. Clinical presentation and imaging of bone and soft-tissue sarcomas. Cleve Clin J Med 2010;77 Suppl 1:S2-7. 